\title{
Success of Different Techniques to Induce Regular Changes in RR-Intervals and Systolic Blood Pressure Levels
}

\author{
EJ Bowers, A Murray \\ Freeman Hospital, Newcastle upon Tyne, UK
}

\begin{abstract}
This study investigated different protocols to induce regular changes in RR-intervals and systolic blood pressure by considering the success of inducing changes, the variability in following the protocols and the variability of the changes.

Twelve subjects had their ECG and continuous noninvasive blood pressure (Finapres) recorded while following four breathing, and four action protocols. All the protocols were designed to induce changes in heart rate and blood pressure at 8 times a minute $(0.133 \mathrm{~Hz})$.

Induction success was $92 \%$ for the breathing protocols and $47 \%$ for the action protocols. Both sets of protocols were performed equally well. With RR-interval changes, variability was 3.1 times lower for breathing protocols than action protocols, and for SP level changes it was 3.4 times lower.

Breathing protocols more successfully induced changes in RR-intervals and SP levels.
\end{abstract}

\section{Introduction}

Baroreflex sensitivity (BRS) was first measured by inducing a sudden change in blood pressure by administrating angiotensin [1]. Since this time various other methods have been used to induce sudden changes in blood pressure or heart rate such as the Valsalava manoeuvre [2] and neck pressure [3]. All these techniques measure BRS in extreme physiological conditions. To measure BRS in more normal physiological conditions, techniques have been developed which use spontaneous variations in heart rate and blood pressure [4][5]. Using the spontaneous techniques, frequency of change cannot be controlled and rate of change has been shown to affect the baroreflex interaction [6].

Regular breathing, which induces regular changes in RR-intervals and systolic blood pressure (SP) levels, has been proposed as a more repeatable way of assessing BRS [7]. Other techniques may also be developed to induce regular changes in RR-intervals and SP levels since hand grip, posture, and mental arithmetic have been shown to effect BRS measurements [8].
This study investigated the success of four different breathing protocols, regular hand grip exercises, rocking, posture changes and mental arithmetic tasks, at inducing regular changes, the variability in following the protocols and the variability of the induced changes.

\section{Methods}

\subsection{Subjects}

Twelve subjects $(30+/-6$ years $)$ had their ECG and continuous non-invasive blood pressure (Finapres) recorded during all protocols.

\subsection{Recording protocols}

All eight protocols were designed to induce changes in RR-intervals and SP levels at 8 times per minute $(0.133 \mathrm{~Hz})$. The protocols were divided into two sets, breathing-induced and action-induced.

Breathing-induced protocols comprised regularly breathing in four different situations: supine, standing, breathing through a resistance, and breathing into a closed orifice. All four protocols gave the subjects visual feedback, so they could adjust either their flow (when breathing supine, standing or through a resistance) or pressure (when breathing into a closed orifice) according to a scrolling signal on an oscilloscope.

Action-induced protocols comprised regularly clenching a fist, being rocked through $\pm 20^{\circ}$, performing mental arithmetic, and having legs regularly raised and lowered. The hand grip protocol gave the subjects visual feedback so the subjects could adjust the force of the hand grip. Subjects were asked to relax the muscles not involved in hand grip as much as possible. Mental arithmetic required indicating whether the equations were true or false, and this was done by finger signals rather than speaking. All subjects answered the mental arithmetic promptly. The protocols involving rocking and having legs raised and lowered were performed by the same operator to minimise operator variability.

All protocols lasted 3 minutes and had 24 cyclic changes. 


\subsection{Analysis}

Induction success was determined by the presence of peaks at $0.133 \mathrm{~Hz}$ in the frequency spectra of the RR-interval and SP level signals. A peak was considered to exist if it was 5 standard deviations greater than the mean value of the signal between 0.1 to $0.5 \mathrm{~Hz}$.

The variability in following the protocols, and of the changes of RR-intervals or SP levels, were calculated by averaging signals over the 24 cycles for each subject and protocol, and establishing the $95 \%$ confidence range, which was then normalised by dividing by the average change. This meant the lower the variability in following the protocol, the lower the index, hence the better the protocol was followed. Similarly the lower the variability of the induced changes, the lower the index, hence the more successful the protocol.

The analysis steps to calculate the variability in following one protocol are shown in figure 1. Figure 1a shows each of the 24 periods of the breathing signal superimposed. Figure 1b shows the average breathing signal over the 24 periods, and from this signal the average change can be calculated, A. Figure 1c shows the mean signal and the $95 \%$ confidence interval at each point along the time scale. Figure 1d shows the averaged $95 \%$ confidence range, $\mathrm{B}$, across all the points calculated from the data in figure $1 \mathrm{c}$. The lower the variability index $(\mathrm{B} / \mathrm{A})$ the better the protocol is performed.

\section{Results}

\subsection{Induction success}

Induction success was $94 \%$ for RR-interval changes and $92 \%$ for SP level changes for the breathing protocols and $46 \%$ and $48 \%$ respectively for the action protocols. As shown in figure 2 there was no significant difference between induction success of RR-interval and SP level changes within protocols $(\mathrm{p}=0.73$ breathing and $\mathrm{p}=0.94$ action). There was also no significant difference between RR-interval and SP-level changes across the protocols ( $p=0.08$ RR-interval and $p=0.12$ SP level).

\subsection{Variability in following protocols}

The variability in following the protocols was good for both sets of protocols. For the breathing protocols the $95 \%$ confidence range was only 0.4 times the signal amplitude (variability index $=40 \%$ ). For the action protocols the $95 \%$ confidence range was only 0.3 times the signal amplitude (variability index $=29 \%$ ). There was no significant difference as shown in figure 3 . This means both sets of protocols were performed equally well.
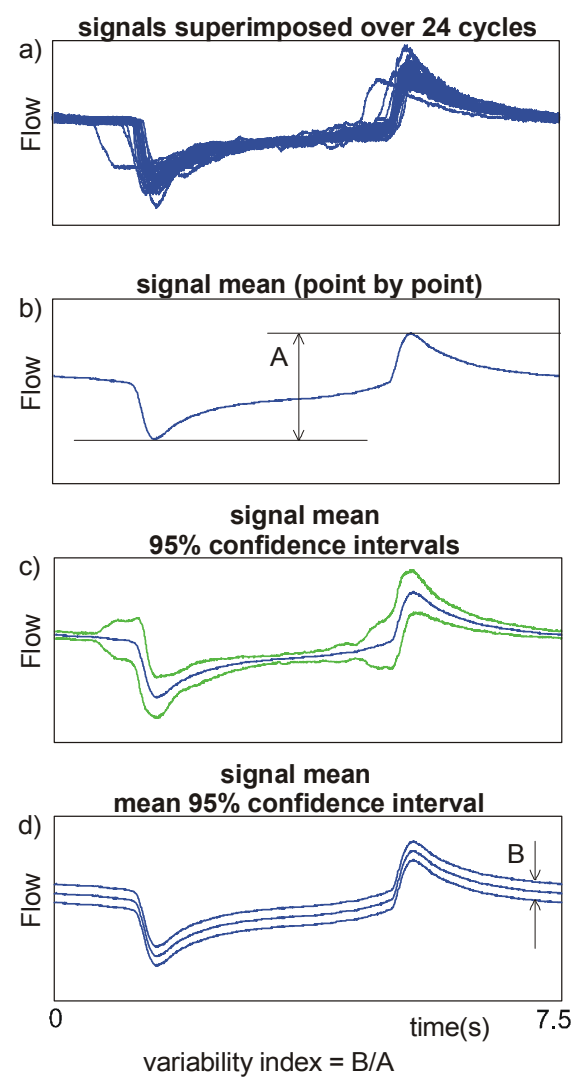

Figure 1 . The analysis steps to calculate the variability in following the protocol. This is shown for a flow signal but the pressure signal from the closed orifice and force signal from the hand grip were used as well. The same analysis techniques were applied to RR-interval and SP level data to calculate the variability of the changes.

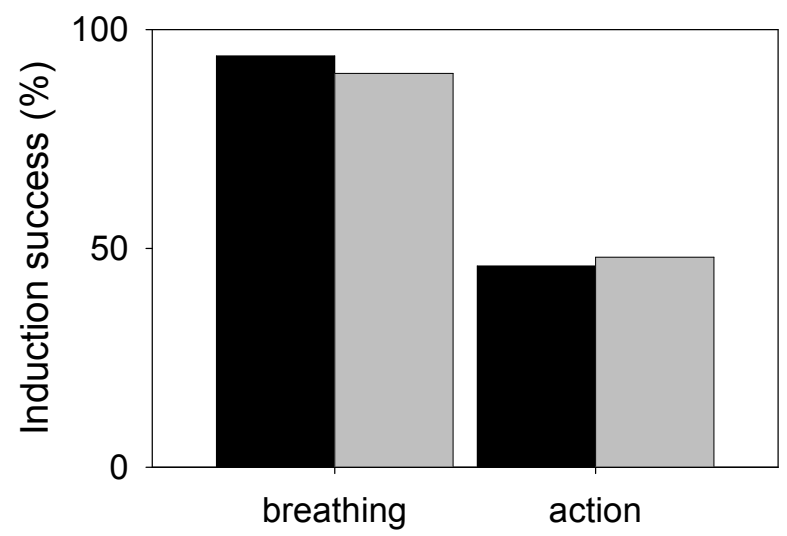

Figure 2: The induction success for RR-interval changes (black bars) and for SP level changes (grey bars). 


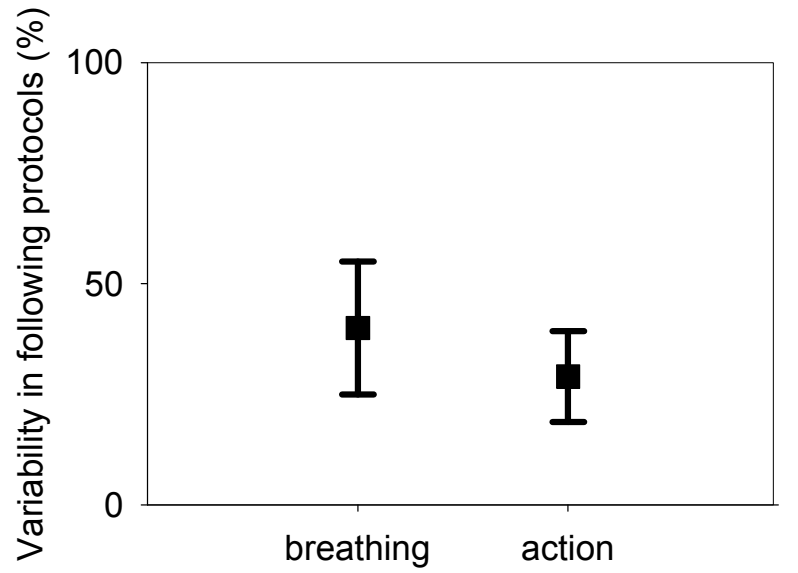

Figure 3. The variability index for following both sets of protocols (mean \pm standard deviation). There was no significant difference in the performance of either set of protocols.

\subsection{Variability of induced changes}

Variability of induced changes was lowest amongst the breathing methods as shown in figure 4. Breathing protocols had a $95 \%$ confidence range that was 1.4 times greater than the change in RR-interval (variability index $=142 \%$ ) and 2.2 times greater than the change in SP levels (variability index $=218 \%$ ). For the action protocols the values were 4.4 and 7.5 respectively (variability index $=440 \%$ and $748 \%$ respectively).

Variability of induced changes for RR-interval was 3.1 times lower for breathing protocols than action protocols, and for SP level changes it was 3.4 times lower. The variability of induced changes for breathing protocols was significantly lower $(p<0.001)$ for both changes in RR-intervals and SP levels.

\section{Discussion}

Breathing protocols were more successful at inducing regular changes in RR-intervals and SP levels. The variability in following the protocols was low, but the variability of the changes of RR-intervals and SP levels were much higher, indicating that other factors significantly influence these changes.

The reason why the variability of the changes of RR-interval and SP levels for the breathing protocols is smaller than that of the action protocols is because the average amplitude of RR-interval and SP levels (A) is larger.

A study by Iellamo [8] considered the repeatability of BRS across two days for normal subjects when breathing normally in a supine position, breathing normally in a standing position, maintaining a static hand grip and performing mental arithmetic. We cannot directly
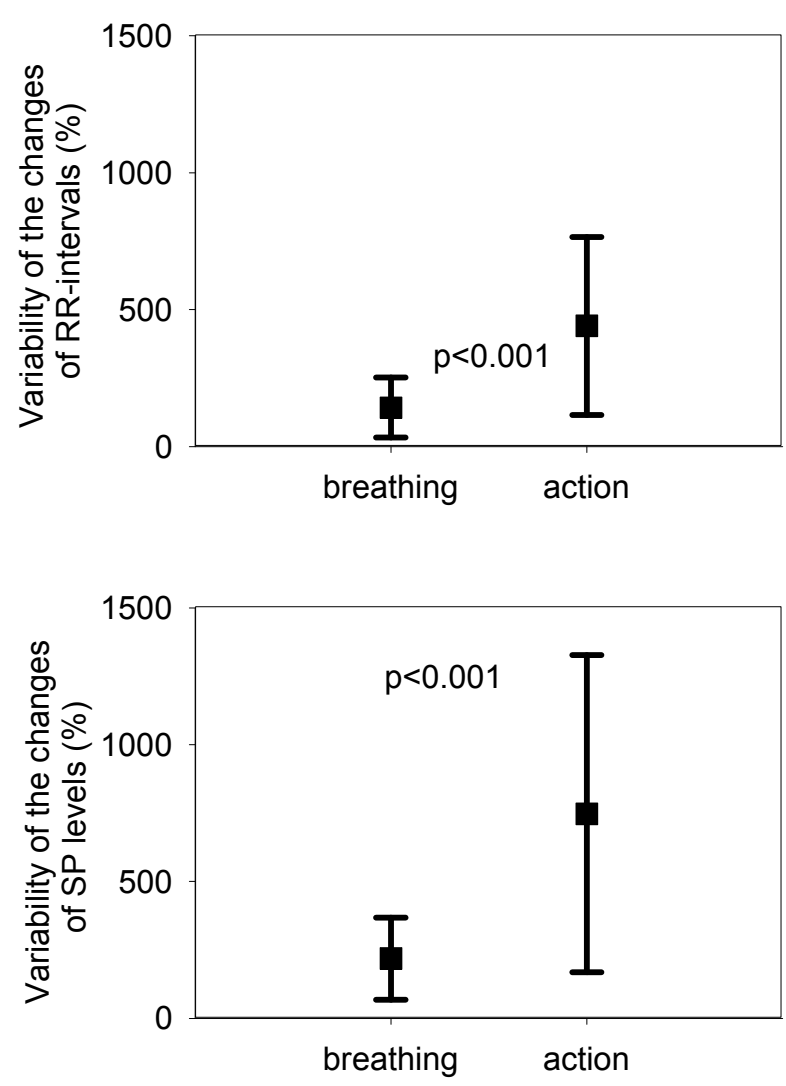

Figure 4. The variability index for RR-intervals and SP levels (mean \pm standard deviation).

compare the study by Iellamo with this study since we are inducing changes at one frequency and we do not calculate BRS. We could hypothesise that the smaller the variation in induced changes in RR-intervals and SP levels the more repeatable the BRS. Iellamo however failed to find a significant difference in repeatability between the four protocols.

When looking at the frequency content of RR-intervals and SP levels for normal breathing there tends to be two peaks: one centred between 0.1 to $0.15 \mathrm{~Hz}$ (the low frequency (LF) peak), and one centred on the average breathing rate (the high frequency (HF) peak) [9]. If breathing is very regular then the height of the LF peak reduces and the height of the HF peak increases; the HF peak also becomes increasingly narrow. It is not fully understood how these two peaks interact especially when the frequency of the regular breathing is close to $0.1 \mathrm{~Hz}$. The induction rate was limited by the ability to raise and lower legs and to tilt patients through $\pm 20^{\circ}$, therefore it should be acknowledged that there may be some unknown interaction between the induced peak and the LF peak which may contribute to the variability of the changes of RR-intervals and SP levels. 
There are two further reasons for the variability figures being high, a) no correction has been made for the variation in mean RR-interval and SP level over the recording time and $b$ ) the variability of change has still been calculated even when the induction success was zero which would skew the results.

This study has considered the worse case of variability on a cycle by cycle level. This shows that breathing protocols induce changes which are less variable than action protocols even though each set of protocols is followed equally well.

\section{Acknowledgements}

This work was funded by The Newcastle upon Tyne Hospitals NHS Charity.

\section{References}

[1] Smyth HS, Sleight P, Pickering GW. Reflex regulation of arterial pressure during sleep in man: a quantitative method of assessing baroreflex sensitivity. Circulation Research 1969;24:109-121.

[2] Korner PI, Tonkin AM, Uther JB. Valsalva constrictor and heart rate reflexes in subject with essential hypertension and with normal blood pressure. Clinical and Experimental Pharmacology and Physiology 1979;6:97-110

[3] Sprenkle JM, Eckberg DL, Goble RL, Schelhorn JJ, Halliday HC. Device for rapid quantification of human carotid baroreceptor-cardiac reflex responses. Journal of Applied Physiology 1986;60:727-732.

[4] Bertinieri G, di Rienzo M, Cavallazzi A, Ferrari AU, Pedotti A, Mancia G. A new approach to analysis of arterial baroreflex. Journal of Hypertension 1985;3:S79S81.
[5] Robbe HWJ, Mulder LJM, Ruddel H, Langewitz WA Veldman JBP, Mulder G. Assessment of baroreceptor reflex sensitivity by means of spectral analysis. Hypertension 1987;10:538-543.

[6] Pitzalis MV, Mastropasqua F, Massari F, Passantino A, Colombo R, Mannarini A, et al. Effect of respiratory rate on the relationship between RR interval and systolic blood pressure fluctuations: a frequency-dependent phenomenon. Cardiovascular Research 1998;38:332-339.

[7] Davies LC, Francis DP, Jurak P, Kara T, Piepoli M, Coats AJ. Reproducibility of methods for assessing baroreflex sensitivity in normal controls and in patients with chronic heart failure. Clinical Science 1999;97:515-522.

[8] Iellamo F, Legramante J, Raimondi G, Castrucci F, Massaro M, Peruzzi G. Evaluation of reproducibility of spontaneous baroreflex sensitivity at rest and during laboratory tests. Journal of Hypertension 1996;14:10991104.

[9] Akselrod S, Gordon D, Madwed JB, Snidman NC, Shannon DC, Cohen RJ. Hemodynamic regulation: investigation by spectral analysis. American Journal of Physiology (Heart and Circulation) 1985;249:H867-H875.

Address for correspondence.

Emma J Bowers

Regional Medical Physics Department

Freeman Hospital

Freeman Road

High Heaton

Newcastle upon Tyne

NE7 7DN

emma.bowers@nuth.northy.nhs.uk 